\title{
Quantitative phase-digital holographic microscopy: a new imaging modality to identify original cellular biomarkers of diseases
}

\author{
P. Marquet*a,b,c, K. Rothenfusser ${ }^{\text {b,c }}$, B. Rappaz ${ }^{\text {d }}$, C. Depeursinge ${ }^{\text {c,e }}$, P. Jourdain ${ }^{\text {b,c }}$, P. J. \\ Magistretti ${ }^{\mathrm{b}, \mathrm{c}, \mathrm{e}}$, \\ ${ }^{a}$ Institut Universitaire en Santé Mentale de Québec, Quebec, Quebec G1J 2G3, Canada, Department \\ of Psychiatry and Neuroscience, Université Laval, Quebec, Quebec G1V 0A6, Canada; ${ }^{b}$ Centre de \\ Neurosciences Psychiatriques, Département de psychiatrie DP-CHUV, Site de Cery, 1008 Prilly- \\ Lausanne, Switzerland ; ${ }^{\mathrm{B}}$ Brain Mind Institute, École Polytechnique Fédérale de Lausanne (EPFL), \\ 1015 Lausanne, Switzerland ; ${ }^{\mathrm{d} B i o m o l e c u l a r}$ Screening Facility (BSF) École Polytechnique Fédérale \\ de Lausanne, CH-1015 Lausanne, Switzerland; ${ }^{\circ}$ Division of Biological and Environmental Sciences \\ and Engineering, King Abdullah University of Science and Technology, Thuwal 23955-6900, \\ Kingdom of Saudi Arabia;
}

\begin{abstract}
Quantitative phase microscopy (QPM) has recently emerged as a powerful label-free technique in the field of living cell imaging allowing to non-invasively measure with a nanometric axial sensitivity cell structure and dynamics. Since the phase retardation of a light wave when transmitted through the observed cells, namely the quantitative phase signal (QPS), is sensitive to both cellular thickness and intracellular refractive index related to the cellular content, its accurate analysis allows to derive various cell parameters and monitor specific cell processes, which are very likely to identify new cell biomarkers. Specifically, quantitative phase-digital holographic microscopy (QP-DHM), thanks to its numerical flexibility facilitating parallelization and automation processes, represents an appealing imaging modality to both identify original cellular biomarkers of diseases as well to explore the underlying pathophysiological processes.
\end{abstract}

Keywords: Digital holographic microscopy, quantitative phase microscopy, cell imaging, cell volume, cell membrane fluctuations, cell biomarkers, high-throughput screening, psychiatry disorders

\section{INTRODUCTION}

During the last decades numerous of QPM techniques based on an interferometer or holographic approaches have been developed $^{1-4}$. Considerably simpler to implement than classical interference microscopy, while providing a reliable and quantitative phase mapping of the observed specimen, they have started to be efficiently used in the field of cell biology. In this section, the main advantages of QP measurement based on a digital holographic microscopy approach, allowing in particular to propagate the whole object wave (amplitude and phase) diffracted by the observed specimen during the numerical reconstruction of the digitally recorded holograms, will be explained in particular as far as living cells are concerned.

\subsection{Quantitative phase-digital holographic microscopy (QP-DHM)}

A digital holographic microscope comprises both an optical setup devoted to hologram formation and software specially developed to numerically process the digitized hologram. The hologram results from the interference of the object beam with a reference wave that can be kept separate from the object beam. The goal is to estimate precisely the propagated wavefront corresponding to either the virtual or the real image of the specimen. A typical DHM arrangement, we have developed and used to explore transparent specimens including living cells, by quantitatively monitoring the phase retardation of the object wave when transmitted through the observed specimen, is a Mach Zehnder interferometer in an off axis geometry ${ }^{5}$.

Quantitative Phase Imaging II, edited by Gabriel Popescu, YongKeun Park, Proc. of SPIE Vol. 9718, 97180K (C) 2016 SPIE $\cdot$ CCC code: $1605-7422 / 16 / \$ 18 \cdot$ doi: $10.1117 / 12.2213454$ 
(a)

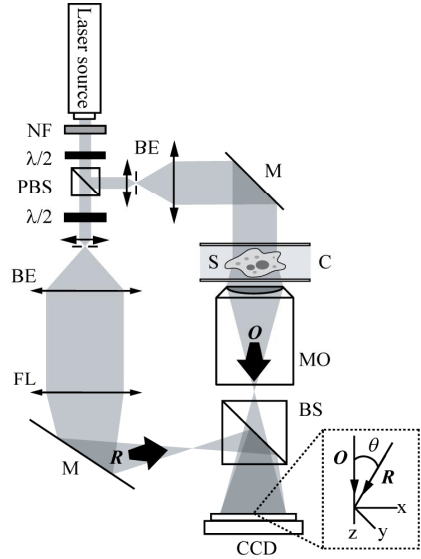

(c)

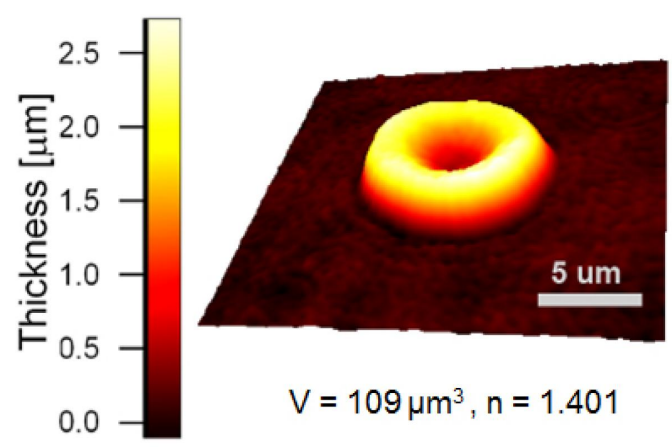

(b)

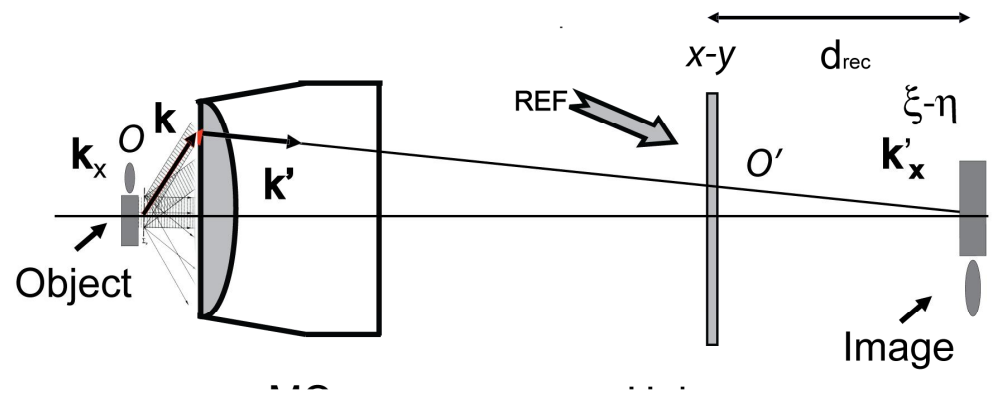

(d)

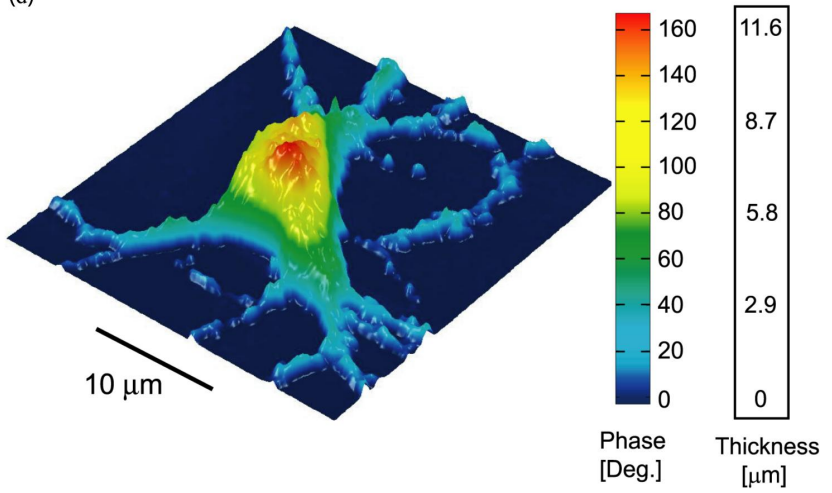

Figure 1. (a) transmission optical setup for QP-DHM (b) role of the microscope objective (MO): magnifying the image of the specimen but also, in terms of wavevectors, decreasing the lateral wavevector component kx or y by a factor proportional the magnification (M) of the MO, so that the hologram can be sampled by an electronic camera according to Shannon's rules (c,d) 3D perspective images in false colors of a living human red blood cell and a mouse cortical neuron in culture respectively. Each pixel represents a quantitative measurement of the phase retardation or cellular optical path length (OPL) induces by the cell with a sensitivity corresponding to a few tens of nanometers. By taking account the measured mean values of the RBC and neuronal cell body RIs, resulting from the decoupling procedure (cf. below), scales (at right), which relate OPL (Deg) to morphology in the z-axis $(\mu \mathrm{m})$, can be constructed.

Abbreviations: BS, beam splitter; $\mathrm{CCD}$, camera; M1 and M2, mirrors; MO, microscope objective; O, object beam; R, reference beam; RL, reference lens and OC, object condenser; object or specimen

An important issue is the need for a reference beam that is controllable in both in intensity and polarization, thus enabling to improve contrast and signal. The holographic principle also permits other valuable concepts building on in particular the possibility of superimposing several holograms. Reference waves corresponding to different wavelengths can also be generated, permitting the use of synthetic wavelengths from a single hologram ${ }^{6}$

As in other developments in digital holography, the hologram reconstruction method in DHM is based on the theory of diffraction. When a MO is used and the specimen can be maintained in the MO's focal plane, the image is formed at infinity. The hologram at infinity is simply the Fourier transform of the specimen. Inverse Fourier transformation straightforwardly provides the reconstructed wavefront in the focal plane of the MO. However, specimens generally are not fully contained in the focal plane of the $\mathrm{MO}$, thus a real and/or virtual image is formed at a finite distance from the MO (Fig. 1 b). 
Intercepting the wavefront at a finite distance from the specimen image gives rise to holograms in the Fresnel zone. Thus, the Huygens-Fresnel expression of diffraction can be used to calculate the propagation of the reconstructed wave. It should be noted that the MO introduced in the interferometer allows to adapt the wavefield to the sampling capacity of the camera. Indeed, the lateral components of the wavevector $\mathrm{k}_{\mathrm{x} \text { or } \mathrm{y}}$ can be divided by the magnification factor $\mathrm{M}$ of the MO. Taking advantage of a full digital approach, a computer can perform the reconstruction by simulating the reference wave and multiplying it by the hologram intensity distribution. Then, the distribution of the wavefront in space is obtained by simulating the propagation of the wavefront reconstructed in the plane of the hologram. Practically, the wavefront gives rise to several propagated beams, the zero-order beam and higher-order beams with diffraction orders of +1 and -1 mainly. When a MO is used, the latter two orders give rise to virtual and real images respectively that appear as twin images, which are the reflection of one another on a virtual mirror that takes the place of the hologram plane.

The Off-axis geometry introduces a spatial carrier frequency, and demodulation restores the full spatial frequency content of the wavefront. The main advantage of this approach is that all the information for reconstructing the complex wavefield comes from a single hologram ${ }^{7}$. In microscopy, the full bandwidth of the beam delivered by the MO can be acquired without limitation. Scale adjustments can make to preserve the correct sampling of the MO's full bandwidth, so that no loss of resolution is conceded. Finally, selecting in the Fourier domain of the hologram the signal corresponding to diffraction order of +1 allows for the full restoration of the object wavefront transmitted by the MO. Reconstructing the wavefront in 3D is therefore simply done by propagating the wavefront generated in the hologram plane $x-y$ to the image plane $\xi-\eta$, which is situated at a distance $d_{\text {rec }}$. This could be efficiently achieved by computing the Fresnel transform of the wavefield. DHM brings thus quantitative data derived simultaneously from the amplitude and the phase of the complex reconstructed wavefront that is diffracted by the object. Our method requires the adjustment of several reconstruction parameters which can be done using a computer-aided method we developed. Some image processing is also needed to improve the accuracy of the reconstructed phase ${ }^{8}$. A priory any type of aberrations can also be corrected during such a numerical reconstruction process ${ }^{9,10}$. Using a high numerical aperture, submicron transverse resolution has been achieved to 300-nm lateral resolution. Accuracy of approximately $0.1^{\circ}$ have been estimated for phase measurements. In reflection geometry, this corresponds to a vertical resolution of less than $1 \mathrm{~nm}$ at a wavelength of 632 $\mathrm{nm}{ }^{11}$. In transmission geometry, the resolution is limited to a few nanometers as far as living cells are concerned. It is also worthwhile to note that when the experimental configuration of the hologram recording requires distances of reconstruction for which the Fresnel approximation does not properly work, the propagation of the wavefront generated by the hologram can be efficiently performed with the angular spectrum approach ${ }^{12,13}$.

\section{CELL IMAGING WITH QUANTITATIVE PHASE MICROSCOPY (QPM)}

Biological specimens such as living cells and tissues are usually phase objects, that is, they are transparent and made visible most often by $\mathrm{PhC}$ as explained above. The phase signal originates in the RI difference generated by the presence of organic molecules in cells, including proteins, DNA, organelles, and nuclei. Consequently, QPM visualizes cells by quantitatively providing the phase retardation that they induce on the transmitted wavefront ${ }^{5}$. 3D perspective images of such a phase retardation corresponding to a living human red blood cell and a mouse cortical neuron in culture are shown on Fig. 1c, d respectively. This QPS signal is given by the following expression:

$$
\varphi=\frac{2 \pi}{\lambda}\left(\bar{n}_{c}-n_{m}\right) d
$$

where $d$ is the cellular thickness, $\bar{n}_{c}$ is the intracellular RI averaged over the OPL of optical rays crossing the specimen, and $n_{m}$ is the RI of the surrounding medium. The cellular quantitative phase contrast, results thus from the fact that the intracellular RI is different from the RI of the perfusion solution $n_{m}$.

As indicated by the Eq. 1, QPS contains information about the cell morphology. It results that QPS can be used to efficiently perform cell counting, recognition and classification analysis ${ }^{2,14}$. Specifically, QP-DHM allowing, thanks to the numerical propagation of the reconstructed object wavefront, to apply autofocusing and extended depth of focus has opened the possibility of efficiently monitoring cell movements and migration in $3 \mathrm{D}^{14}$, in particular when microfluidic 
devices are considered ${ }^{15}$. Since the QPS is also sensitive to intracellular RI, it specifically provides some information about the intracellular content. Based on this information, interesting applications allowing discrimination between physiological and pathophysiological states have been achieved ${ }^{2}$

\section{HIGH CONTENT PHENOTYPIC SCREENING BASED ON QP-DHM}

High content screening (HCS $)^{16}$ is widely used today in academic and pharmaceutical research for investigating a large variety of biological processes through the design phenotypic cellular assays (i.e. based on morphological changes). A series of important screening applications in biological or therapeutic research target the measurement of cell morphological variations ${ }^{17}$ for monitoring events such as cell death mechanisms and cytotoxicity profiling upon interaction with drugs or interfering compounds. For these applications, it is of paramount importance to use the least invasive imaging techniques possible to avoid phenotypic changes induced by the imaging process itself. Therefore noninvasive and label-free technologies offer a strong advantage in comparison with labeled approaches for which the requirement of exogenous labels may alter the intactness of cells, besides the need for extra pipetting or dispensing steps to deliver probes and the required time-consuming image focusing process prior to image acquisition, which are particularly limiting as far as time lapse experiments are concerned. As illustrated by the different applications presented above, the QPS provides original and unique information about cell morphology and content with a high sensitivity. Consequently, QPM in general and in particular QP-DHM, thanks to its numerical flexibility, represent an appealing imaging modality to perform high content phenotypic screening.. This view is supported by some studies which have permitted to stress the possibility to develop in vitro label-free cytotoxicity screening assays based on QP-DHM ${ }^{18-21}$. Specifically, Pavillon et al. ${ }^{21}$ have explored in vitro the early detection of excitatory neuronal death induced by perfusion of glutamate, the main excitatory neurotransmitter in the brain (cf. section 4.2). Practically, the analysis of the early QPS changes, reflecting in particular a rapid neuronal cell volume regulation in response to glutamate, has permitted to predict within a time frame of tens of minutes, whether a subsequent neuronal death would occur. Otherwise, HCS technology requires to be both high in content and in screening and thus consists in the parallelization and automation of a test normally conducted in a single petri dish. For this purpose 96,384 or 1536 well plates in which cells are cultured (mostly cell lines or primary cell cultures) are typically used. The compounds to test are added to each well of the plate (before or after adding the cells). Practically, one compound is added per well and a few tens to a few millions compounds can be tested. Concretely, a few images per well are acquired and automated image analysis characterizes and quantifies the morphological cell changes induced by the specific compound presents in each well.

Indeed, although QP-DHM does not have the advantage of signal specificity provided by labeled approaches, it provides a non-invasive highly sensitive QPS allowing to directly quantify cytotoxicity ${ }^{18}$ and permits a drastic improvement in scanning speed for multi-well plates thanks to its numerical autofocusing capability. Practically, instead of having to mechanically focus on each well, a re-focusing step is performed off line after the acquisition during the numerical reconstruction of the holograms, in parallel to the image analysis and quantification.

Otherwise, standard image processing of quantitative phase images allows to rapidly determine which regions of the field contain cells and thus to automatically provide relevant information including cell confluence, proliferation or migration. From that, many parameters derived from the QPS can be defined to quantitatively and specifically characterize the phenotypic cell changes mediated by the tested compounds. For illustrative purpose, Fig. 3 shows a time courses of the mean optical thickness $\left(\mathrm{OT}=(\lambda / 2 \pi)^{*} \mathrm{QPS}\right)$ of a HeLa cell population treated with serial dilution of doxorubicin and the corresponding dose-response graph obtained by measuring the area under the curve of the timelapse date and allowing to calculate the half maximal effective concentration $\left(\mathrm{EC}_{50}\right)$. 

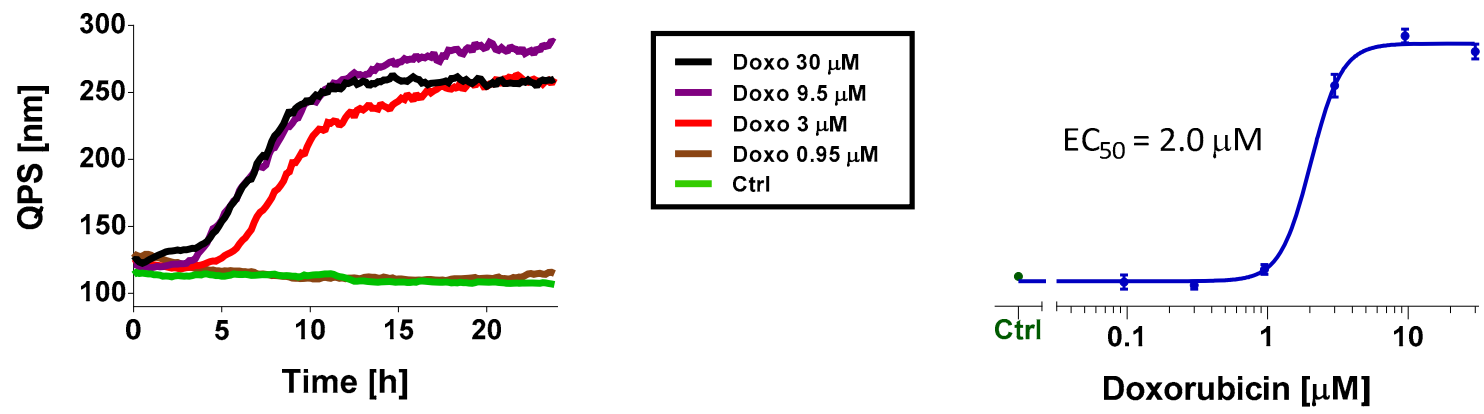

Figure $224 \mathrm{~h}$ time-lapse measurement of the QPS of HeLa cells (a cancer cell line) treated with various concentrations of doxorubicin (an anticancer drug) as well as the corresponding the dose-response graph allowing to derive the halfnaximum effective concentration $\left(\mathrm{EC}_{50}\right) \mathrm{OT}=$ optical thickness defined as the mean $(\lambda / 2 \pi)^{*} \mathrm{QPS}$ of HeLa cell population. Modified from ${ }^{19}$

Furthermore, time-lapses yield more information than simple end-point measurements obtained after a given period of time. For instance, we can detect when cells start to respond to the treatment (4-5 h in the case of doxorubicin), when a plateau is reached (here at $\sim 12 \mathrm{~h}$ ) therefore obtaining a full vision of the dynamics of the process.

In addition to these automated measurements reflecting the behavior of a cell population, a further image analysis aiming at identifying relevant quantitative parameters at a single cell level can be easily performed ${ }^{17}$. This type of analysis allows for instance to distinct subpopulations of cells exhibiting different phenotypes

\section{QP-DHM: A TOOL TO IDENTIFY AND SCREEN NEW CELL BIOMARKERS OF PSYCHIATRIC DISORDERS}

Psychiatric disorders represent major public health problems and, while significant improvements of the conditions of the patients have been achieved thanks to the discovery of neuroleptics and antidepressants, the available treatments remain largely insufficient and unsatisfactory. A major paradigm shift is needed to move the field forward. Advances in the field are impaired in large part due to the lack of proper tools to conduct adequate biological measurements that can guide diagnosis and treatment. This is particularly critical in light of growing evidence showing that early treatment of psychiatric disorders, already in their prodromal phase and before the onset of debilitating symptoms, leads to significantly improved clinical outcomes ${ }^{22}$. Hence, the necessity to already identify in children biomarkers or endophenotypes reflecting a high risk of later developing the disease. However, to date there are insufficient data to support any of these biomarkers as vulnerability, diagnostic or prognostic factors, notably regarding bipolar disorders ${ }^{23}$. In this field of research, the most relevant investigations are longitudinal studies conducted on cohorts consisting of patients with a specific psychiatric disorder and their children (high-risk children). In addition, psychiatric disorders are frequently comorbid with other mental illnesses as well as endocrine, metabolic (e.g., diabetes mellitus and obesity) and cardiovascular diseases. Thus, they must be considered as a multi-system condition, and as a consequence, their underlying neurobiological bases are complex. Coherently, only studies combining several biomarkers have been successful in particular to separate acute mood states (mania and depression) from controls ${ }^{24}$ and to provide a high accuracy for the diagnosis of major depression. Consequently, the identification of such reliable early biomarkers of vulnerability, neuroprogession and diagnostic of mental disorders requires to identify and to longitudinally study various sets of biomarkers very diverse in nature including e.g. neuropsychological, neuroimaging (structural and functional MRI), as well as peripheral biomarkers or cellular biomarkers. Practically, the study of cell biomarkers can be achieved from somatic cell (fibroblasts) obtained from patients as well as from high-risk children (skin biopsy).

Within this framework a multimodality imaging approach developed around QP-DHM has the capacity to explore new, original optical cellular biomarkers down at the nanoscale that can be then put in contrast to other more conventional markers mentioned above ${ }^{14}$. Indeed QP-DHM provides a unique monitoring of fine cell structure and dynamics thanks to 
its ability to yield quantitatively information on a wide variety of cellular parameters including volume, morphology intracellular protein content, dry weight, nanoscale membrane fluctuations, membrane water permeability, transmembrane water movements and volume regulation. The value of such an approach can be illustrated by a study we are conducting in a cohort of diabetic patients aiming at correlating energy distribution among vibrational eigenmodes of Red Blood Cell (RBC) membranes with the levels of glycosylated hemoglobin (Fig. 3).

a)

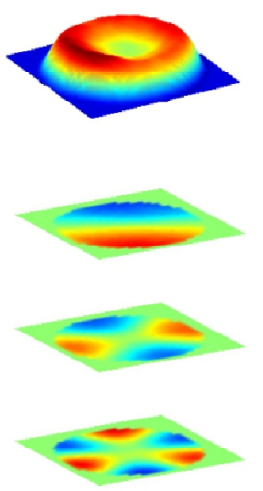

c)

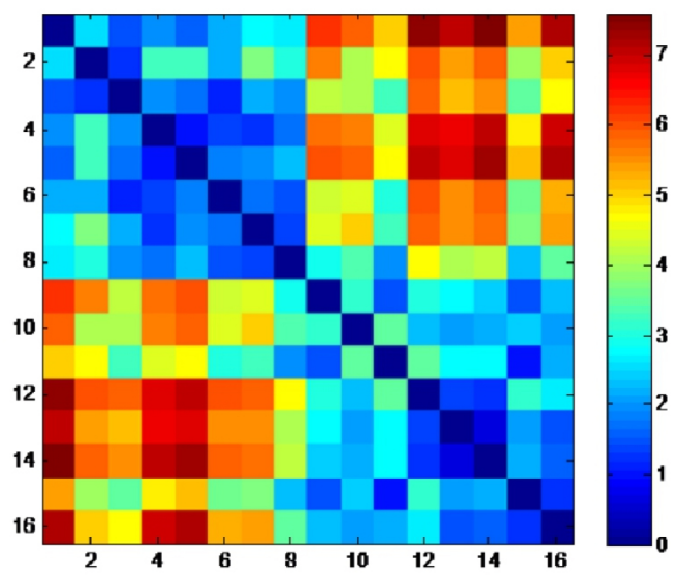

Figure 3 a) Quantitative phase image of a Red Blood Cell (RBC) in a perspective color coded representation and projections of three Principal Component Analysis (PCA) modes of the RBC cell membrane fluctuations. b) Degeneracy free representation of 20 PCA modes, measured experimentally. c) Each t-value (i,j) in the matrix indicates how similar the energy distribution of the PCA modes between subject $\mathrm{i}$ and subject $\mathrm{j}$ are. The lower this $\mathrm{t}$-value, the more similar the energy distribution is. Healthy (1-8) and diabetic (916) samples can be discriminated.

\section{PERSPECTIVES}

QPM and specifically DH-QPM thanks to the unique possibilities offered by the numerical reconstruction and propagation of the object wavefront including extended depth of focus, a posteriori numerical autofocusing, allows to quantitatively and non-invasively study cell structure, dynamics as well as content at different scales of time ranging from a few milliseconds to several days. However, although the QPS is highly sensitive and contains a wealth of information, its interpretation, in terms of specific biological processes remains an issue. Practically, addressing this interpretation issue involves the ability to calculate from the phase signal relevant biophysical cell parameters including absolute volume, membrane fluctuations at the nanoscale, biomechanical properties, transmembrane water permeability and current, etc. Concretely, the derivation of these cell biophysical parameters often requires to separately obtain cell morphology (thickness) and content (intracellular RI). Within this framework, the development of DHM-based optical diffraction tomography, providing a direct access to the 3D map of the intracellular RI could allow to revisit this difficulty of interpretation. On the other, QP-DHM or QPM in multimodality approaches combining e.g. fluorescence ${ }^{25}$, spectroscopy, coherent anti-Stokes Raman scattering (CARS), second (SHG) and third harmonic generation (THG) should allow simultaneous measurements of a large number of relevant and specific parameters yielding an extended cell profiling approach. Specifically, SHG ${ }^{26}$ and $\mathrm{THG}^{27}$ convey highly relevant information concerning in particular membrane proteins and intracellular constituents. Due to the highly non-linear character of these light-generating processes, local properties of cytoskeleton and membrane are obtainable, as well 
as $3 \mathrm{D}$ cellular arrangements by taking advantage of the remarkable penetration power of the SHG or, more particularly, the THG processes. Within this framework, it is worthwhile mentioning one of the first attempts to combine Raman information with quantitative phase information paving thus the way to simultaneously explore the cell molecular composition and dynamics ${ }^{28}$. Otherwise, used in conjunction with AFM, QP-DHM would provide an image of the local deformations and therefore a more detailed and informative insight of biophysical properties of the cell. In addition, the use of free wandering particles would also inform precisely on the nature of binding forces and properties of the macromolecules. This detection of multiple biophysical cell parameters could even be scaled into a high-throughput cellular screening assay ${ }^{18}$ allowing the monitoring of several cellular processes concurrently as well as their modulations as a function of the transcriptome and metabolome of different cell-types derived from controls, patients as well as high-risk subjects. This paves the way to explore cell biomarkers of risk for different diseases. Specifically, within the framework of longitudinal studies conducted on cohorts of patients and their children as previously mentioned, such high-throughput multimodality approaches would be highly promising to identify new and original early cell biomarkers or endophenotypes of mental disorders that will become invaluable tools for early diagnosis, improved stratification, a personalized medicine approach and assessment of disease progression as well as of treatment outcome.

\section{ACKNOWLEDGEMENT}

The work presented in this review is the result of a close collaboration between the Microvision and microdiagnosis group (SCI / STI / CHD group) of EPFL with Florian Charrière, Jonas Kühn, Etienne Shaffer, and Christian Depeursinge, the Laboratory of Neuroenergetics and Cellular Dynamics, Brain and Mind Institute of EPFL with Pierre Magistretti, Benjamin Rappaz, Pascal Jourdain, the Center for Psychiatric neuroscience, CHUV with Daniel Boss, Kaspar Rothenfusser and the company Lyncée Tec SA, Lausanne (http://www.lynceetec.com). We thank the Swiss National Science Foundation (SNSF) grants $n^{\circ}$ CR3213 132993 the National Center of competence in research Synapsy (http://www.nccr-synapsy.ch/) as well as the fondation de Préfargier

\section{REFERENCES}

1 Marquet, P., Depeursinge, C. \& Magistretti, P. J. Exploring Neural Cell Dynamics with Digital Holographic Microscopy. Annual Review of Biomedical Engineering, Vol 15 15, 407-431, doi:DOI 10.1146/annurev-bioeng071812-152356 (2013).

2 Lee, K. et al. Quantitative Phase Imaging Techniques for the Study of Cell Pathophysiology: From Principles to Applications. Sensors-Basel 13, 4170-4191, doi:Doi 10.3390/S130404170 (2013).

3 Mico, V., Garcia, J., Zalevsky, Z. \& Javidi, B. Phase-Shifting Gabor Holographic Microscopy. J Disp Technol 6, 484-489, doi:10.1109/jdt.2010.2041526 (2010).

4 Popescu, G. \& Park, Y. Quantitative phase imaging in biomedicine. J Biomed Opt 20, 111201, doi:10.1117/1.JBO.20.11.111201 (2015).

5 Marquet, P. et al. Digital holographic microscopy: a noninvasive contrast imaging technique allowing quantitative visualization of living cells with subwavelength axial accuracy. Opt Lett 30, 468-470 (2005).

6 Kuhn, J. et al. Real-time dual-wavelength digital holographic microscopy with a single hologram acquisition. Opt Express 15, 7231-7242, doi:10.1364/oe.15.007231 (2007).

7 Cuche, E., Marquet, P. \& Depeursinge, C. Simultaneous amplitude-contrast and quantitative phase-contrast microscopy by numerical reconstruction of Fresnel off-axis holograms. Appl Optics 38, 6994-7001, doi:Doi 10.1364/Ao.38.006994 (1999).

8 Cuche, E., Marquet, P. \& Depeursinge, C. Spatial filtering for zero-order and twin-image elimination in digital off-axis holography. Appl Optics 39, 4070-4075, doi:10.1364/ao.39.004070 (2000).

9 Colomb, T. et al. Automatic procedure for aberration compensation in digital holographic microscopy and application to specimen shape compensation. . Appl Opt 45, 851-863 (2006).

10 Colomb, T. et al. Total aberrations compensation in digital holographic microscopy with a reference conjugated hologram. Opt Express 14, 4300-4306 (2006). 
11 Kuhn, J. et al. Axial sub-nanometer accuracy in digital holographic microscopy. Meas Sci Technol 19, doi:10.1088/0957-0233/19/7/074007 (2008).

12 Kim, M. K., Yu, L. \& Mann, C. J. Interference techniques in digital holography. Journal of Optics a-Pure and Applied Optics 8, S518-S523 (2006).

13 Kemper, B. et al. Investigation of living pancreas tumor cells by digital holographic microscopy. J Biomed Opt 11, doi:10.1117/1.2204609 (2006).

14 Marquet, P., Depeursing, C. \& Magistretti, P. Review of quantitative phase-digital holographic microscopy: promising novel imaging technique to resolve neuronal network activity and identify cellular biomarkers of psychiatric disorders. Neurophotonics 1 (2014).

15 Paturzo, M. et al. Microscopy imaging and quantitative phase contrast mapping in turbid microfluidic channels by digital holography. Lab Chip 12, 3073-3076, doi:Doi 10.1039/C2lc40114b (2012).

16 Bickle, M. The beautiful cell: high-content screening in drug discovery. Analytical and Bioanalytical Chemistry 398, 219-226, doi:DOI 10.1007/s00216-010-3788-3 (2010).

17 Carpenter, A. E. et al. CellProfiler: image analysis software for identifying and quantifying cell phenotypes. Genome Biol 7, doi:Artn R100 Doi 10.1186/Gb-2006-7-10-R100 (2006).

18 Kuhn, J. et al. Label-free cytotoxicity screening assay by digital holographic microscopy. Assay and drug development technologies 11, 101-107, doi:10.1089/adt.2012.476 (2013).

19 Rappaz, B., Breton, B., Shaffer, E. \& Turcatti, G. Digital holographic microscopy: a quantitative label-free microscopy technique for phenotypic screening. Combinatorial chemistry \& high throughput screening 17, 8088 (2014).

20 Bauwens, A. et al. Differential cytotoxic actions of Shiga toxin 1 and Shiga toxin 2 on microvascular and macrovascular endothelial cells. Thrombosis and haemostasis 105, 515-528, doi:10.1160/TH10-02-0140 (2011).

21 Pavillon, N. et al. Early Cell Death Detection with Digital Holographic Microscopy. Plos One 7, e30912, doi:ARTN e30912 DOI 10.1371/journal.pone.0030912 (2012).

22 Berk, M. et al. From neuroprogression to neuroprotection: implications for clinical care. The Medical journal of Australia 193, S36-40 (2010).

23 Luby, J. L. \& Navsaria, N. Pediatric bipolar disorder: evidence for prodromal states and early markers. Journal of child psychology and psychiatry, and allied disciplines 51, 459-471, doi:10.1111/j.1469-7610.2010.02210.x (2010).

24 Kapczinski, F. et al. Peripheral biomarkers and illness activity in bipolar disorder. Journal of psychiatric research 45, 156-161, doi:10.1016/j.jpsychires.2010.05.015 (2011).

25 Pavillon, N. et al. Cell morphology and intracellular ionic homeostasis explored with a multimodal approach combining epifluorescence and digital holographic microscopy. J Biophotonics 3, 432-436, doi:DOI 10.1002/jbio.201000018 (2010).

26 Campagnola, P. Second harmonic generation imaging microscopy: applications to diseases diagnostics. Anal Chem 83, 3224-3231, doi:10.1021/ac1032325 (2011).

27 Fujita, K. \& Smith, N. I. Label-free molecular imaging of living cells. Molecules and cells 26, 530-535 (2008).

28 Pavillon, N., Hobro, A. J. \& Smith, N. I. Cell optical density and molecular composition revealed by simultaneous multimodal label-free imaging. Biophys J 105, 1123-1132, doi:10.1016/j.bpj.2013.07.031 (2013). 